\title{
Solving Fuzzy MCDM by Subtracting Benefit Criteria from Cost Criteria
}

\author{
Ta-Chung Chu \\ Department of Management and Information Technology, Southern Taiwan University of Science and Technology, Taiwan
}

Copyright (C) 2015 by authors, all rights reserved. Authors agree that this article remains permanently open access under the terms of the Creative Commons Attribution License 4.0 International License

\begin{abstract}
This paper suggests a fuzzy MCDM (multiple criteria decision-making) approach, where ratings of alternatives versus criteria and weights of criteria are assessed in fuzzy numbers or linguistic values represented by fuzzy numbers. Criteria are classified into cost and benefit ones. In the proposed method, the ratings assigned by decision makers to each alternative versus each criterion and the weights assigned by decision makers to each criterion are averaged. The averaged cost and benefit ratings are further normalized into comparable scales respectively. The membership function of subtracting the summation of weighted normalized benefit ratings from that of weighted normalized cost ratings for each alternative can be developed by interval arithmetic of fuzzy numbers. The fuzzy number ranking method of centroid is then applied to determine the ordering of the alternatives. A numerical example of robot selection demonstrates feasibility of the proposed method.
\end{abstract}

Keywords Fuzzy MCDM, Cost, Benefit, Interval Arithmetic, Centroid

\section{Introduction}

Fuzzy multiple criteria decision-making (MCDM) is a powerful tool for evaluation and selection of alternatives versus different criteria, where ratings of alternatives under different criteria and the importance weights of criteria are usually assessed in fuzzy numbers or linguistic values (Zadeh, 1975) represented by fuzzy numbers. Numerous fuzzy MCDM methods have been investigated. A review of many of these methods can be found in Carlsson and Fuller (1996), Ribeiro (1996), Chu and Varma (2012), and Moghimi and Anvari (2014). Some other recent methods can be found in (Afkham, et al., 2012; Akdag, et al., 2014; Büyüközkan, et al., 2011; Chung et al., 2015; Ghorbani, et al., 2013; Govindan, et al., 2013). However, clear development for the membership function of the aggregation of the fuzzy weighted ratings of each alternative cannot be found in the above works. This limitation deters their applicability to real world problems.

To resolve the above problem, a new fuzzy MCDM approach for alternative selection is suggested. In this work, criteria are categorized into cost and benefit ones. Cost criteria have the property: the smaller, the better. Conversely, benefit criteria have the property: the larger, the better. Via the proposed method, the ratings given by decision-makers to each alternative under each criterion and the weights given by decision-makers to each of the criteria are averaged. Since criteria may have incommensurable units (Chen and Hwang, 1992), all averaged cost and benefit ratings are further normalized into comparable scales respectively before weighted. Obviously, if the summation of the weighted normalized cost ratings (SWNCR) is smaller and/or the summation of the weighted normalized benefit ratings (SWNBR) is larger, the higher priority the alternative will have. The larger value the SWNBR offsets the SWNCR, the higher priority the alternative will have. Thus, the concept of subtracting SWNBR from SWNCR is used to evaluate the alternative performance (or suitability). If final fuzzy evaluation value is smaller, priority of the alternative is higher. Using interval arithmetic of fuzzy numbers can develop the membership function of subtracting SWNBR from SWNCR for each alternative. Because the final fuzzy evaluation values are still fuzzy numbers, then a ranking method is needed.

Many fuzzy number ranking methods have been proposed since fuzzy set theory was introduced by Zadeh in 1965. A comparison of many ranking methods can be seen in Wang and Kerre (2001) and Wang and Lee (2008). Some recent works can be found in (Asady, 2010; Ezzati, et al., 2012; Hari Ganesh and Jayakumar, 2014; Jafarian and Rezvani, 2013; Rao and Shankar, 2013; Sharma, 2015; Thorani, et al., 2012). Despite the merits, some methods are computational complex and others are difficult to present connection by formula between the ranking procedure and the final fuzzy evaluation values of alternatives under fuzzy MCDM model. To resolve the above limitations, this work applies the centroid method (Yager, 1981) to rank alternatives under fuzzy MCDM. The suggested method provides an extension to the fuzzy MCDM techniques available. A numerical 
example of robot selection demonstrates feasibility of the proposed method.

\section{Fuzzy Numbers}

Definition 1. A real fuzzy number $A$ is described as any fuzzy subset of the real line $R$ with membership function $f_{A}$ which possesses the following properties (Dubois and Prade, 1978):

1. $f_{A}$ is a continuous mapping from $R$ to the closed interval $[0,1]$;

2. $f_{A}(x)=0$, for all $x \in(-\infty, a]$;

3. $f_{A}$ is strictly increasing on $[a, b]$;

4. $f_{A}(x)=1$, for all $x \in[b, c]$;

5. $f_{A}$ is strictly decreasing on $[c, d]$;

6. $f_{A}(x)=0$, for all $x \in[d, \infty)$,

where $a, b, c$ and $d$ are real numbers. We may let $a=-\infty$, or $a=b$, or $b=c$, or $c=d$, or $d=+\infty$. Unless elsewhere specified, it is assumed that $A$ is convex, normal and bounded, i.e. $-\infty<a, d<\infty$.

The membership function $f_{A}$ of the fuzzy number $A$ can also be expressed as:

$$
f_{A}(x)= \begin{cases}f_{A}^{\mathrm{L}}(x), & a \leq x \leq b \\ 1, & b \leq x \leq c \\ f_{A}^{\mathrm{R}}(x), & c \leq x \leq d \\ 0, & \text { otherwise }\end{cases}
$$

where $f_{A}^{\mathrm{L}}(x)$ and $f_{A}^{\mathrm{R}}(x)$ are the left and right membership functions of fuzzy number $A$ respectively.

The fuzzy number $A$ is a triangular fuzzy numbers if its membership function $f_{A}$ is given by (Laarhoven and Pedrycz, 1983):

$$
f_{A}(x)= \begin{cases}(x-a) /(b-a), & a \leq x \leq b \\ (x-c) /(b-c), & b \leq x \leq c \\ 0, & \text { otherwise }\end{cases}
$$

where $a, b$ and $c$ are real numbers.

Definition 2. The $\alpha$-cut of fuzzy number $A$ can be defined as (Kaufmann and Gupta, 1991)

$$
A^{\alpha}=\left\{x \mid f_{A}(x) \geq \alpha,\right\} \text {, where } x \in R, \alpha \in[0,1] .
$$

$A^{\alpha}$ is a non-empty bounded closed interval contained in $R$ and it can be denoted by $A^{\alpha}=\left[A_{l}^{\alpha}, A_{u}^{\alpha}\right]$, where $A_{l}^{\alpha}$ and $A_{u}^{\alpha}$ are the lower and upper bounds of the closed interval respectively. For example, if triangular fuzzy number $A=(a, b, c)$, then the $\alpha$-cut of $A$ can be expressed as:

$$
A^{\alpha}=\left[A_{l}^{\alpha}, A_{u}^{\alpha}\right]=[(b-a) \alpha+a,(b-c) \alpha+c]
$$

Given fuzzy numbers $A$ and $B, A, B \in R^{+}$, the $\alpha$-cuts of $A$ and $B$ are $A^{\alpha}=\left[A_{l}^{\alpha}, A_{u}^{\alpha}\right]$ and $B^{\alpha}=\left[B_{l}^{\alpha}, B_{u}^{\alpha}\right]$, respectively. By the interval arithmetic, some main operations of $A$ and $B$ can be expressed as follows (Kaufmann and Gupta, 1991):

$$
\begin{gathered}
(A \oplus B)^{\alpha}=\left\lfloor A_{l}^{\alpha}+B_{l}^{\alpha}, A_{u}^{\alpha}+B_{u}^{\alpha}\right\rfloor, \\
(A \ominus B)^{\alpha}=\left\lfloor A_{l}^{\alpha}-B_{u}^{\alpha}, A_{u}^{\alpha}-B_{l}^{\alpha}\right\rfloor \\
(A \otimes B)^{\alpha}=\left[A_{l}^{\alpha} \cdot B_{l}^{\alpha}, A_{u}^{\alpha} \cdot B_{u}^{\alpha}\right], \\
(A \oslash B)^{\alpha}=\left[\frac{A_{l}^{\alpha}}{B_{u}^{\alpha}}, \frac{A_{u}^{\alpha}}{B_{l}^{\alpha}}\right] \\
(A \otimes r)^{\alpha}=\left\lfloor A_{l}^{\alpha} \cdot r, A_{u}^{\alpha} \cdot r\right\rfloor, r \in R^{+} .
\end{gathered}
$$

\section{A New Fuzzy MCDM Approach}

Assume that a committee of $k$ decision-makers (i.e., $D_{1}, D_{2}, \ldots, D_{k}$ ) is responsible for evaluating $m$ alternatives (i.e., $\left.A_{1}, A_{2}, \ldots, A_{m}\right)$ under $n$ selection criteria (i.e., $\left.C_{1}, C_{2}, \ldots, C_{n}\right)$, where the ratings of alternatives versus each of the criteria as well as the weights of all criteria are assessed in fuzzy numbers or linguistic values (Zadeh, 1975) represented by triangular fuzzy numbers. Both cost criteria $(C), j=1 \sim h$, and benefit criteria $(B), j=h+1 \sim n$, are considered.

\subsection{Average ratings and Perform Normalization}

Let $x_{i j t}=\left(o_{i j t}, p_{i j t}, q_{i j t}\right), x_{i j t} \in R^{+}, i=1,2, \ldots, m$, $j=1,2, \ldots, n, t=1,2, \ldots, k$, be the rating assigned to alternative $A_{i}$ by decision-maker $D_{t}$ for criterion $C_{j}$. The averaged rating, $x_{i j}=\left(o_{i j}, p_{i j}, q_{i j}\right)$, of alternative $A_{i}$ under criterion $C_{j}$ assessed by the committee of $k$ decision-makers can be evaluated as:

$$
x_{i j}=(1 / k) \otimes\left(x_{i j 1} \oplus x_{i j 2} \oplus \cdots \oplus x_{i j k}\right)
$$

where $o_{i j}=\sum_{t=1}^{k} o_{i j t} / k, p_{i j}=\sum_{t=1}^{k} p_{i j t} / k$,

$q_{i j}=\sum_{t=1}^{k} q_{i j t} / k$

All averaged criteria, both $C$ and $B$, are further normalized by the Chen method (Chen, 2001) into comparable scales 
respectively. This method preserves the property in which the ranges of normalized triangular fuzzy numbers belong to $[0,1]$. The normalization of the averaged ratings, i.e. $x_{i j}$ 's, is as follows:

$\bar{x}_{i j}=\left(\frac{o_{i j}}{q_{j}^{*}}, \frac{p_{i j}}{q_{j}^{*}}, \frac{q_{i j}}{q_{j}^{*}}\right), q_{j}^{*}=\max _{i} q_{i j}, j \in B$ or $j \in C,(10)$

where $\bar{x}_{i j}$ is the normalized value of $x_{i j}$. For convenience, let $\bar{x}_{i j}=\left(r_{i j}, s_{i j}, t_{i j}\right)$.

\subsection{Average Weights}

Let $w_{j t}=\left(a_{j t}, b_{j t}, c_{j t}\right), w_{j t} \in R^{+}, j=1,2, \ldots, n$, $t=1,2, \ldots, k$, be the weight assigned by decision-maker $D_{t}$ to criterion $C_{j}$. The averaged weight, $w_{j}=\left(a_{j}, b_{j}, c_{j}\right)$, of criterion $C_{j}$ assessed by the committee of $k$ decision-makers can be evaluated as:

$$
w_{j}=(1 / k) \otimes\left(w_{j 1} \oplus w_{j 2} \oplus \cdots \oplus w_{j k}\right)
$$

where $a_{j}=\sum_{t=1}^{k} a_{j t} / k, b_{j}=\sum_{t=1}^{k} b_{j t} / k$,

$c_{j}=\sum_{t=1}^{k} c_{j t} / k$

\subsection{Develop Membership Function}

The membership function of subtracting the SWNBR from the SWNCR for each alternative, i.e. $G_{i}=\sum_{j=1}^{h} \bar{x}_{i j} \otimes w_{j}-\sum_{j=h+1}^{n} \bar{x}_{i j} \otimes w_{j}$, can be developed by Eqs. (3) (8) as follows:

$$
\begin{aligned}
\left(\bar{x}_{i j} \otimes w_{j}\right)^{\alpha}= & {\left[\left(s_{i j}-r_{i j}\right)\left(b_{j}-a_{j}\right) \alpha^{2}+\left[r_{i j}\left(b_{j}-a_{j}\right)+a_{j}\left(s_{i j}-r_{i j}\right)\right] \alpha+r_{i j} a_{j},\right.} \\
& \left.\left(s_{i j}-t_{i j}\right)\left(b_{j}-c_{j}\right) \alpha^{2}+\left[t_{i j}\left(b_{j}-c_{j}\right)+c_{j}\left(s_{i j}-t_{i j}\right)\right] \alpha+t_{i j} c_{j}\right]
\end{aligned}
$$

Thus, we have

$$
\begin{gathered}
G_{i}^{\alpha}=\left(\sum_{j=1}^{h} \bar{x}_{i j} \otimes w_{j}-\sum_{j=h+1}^{n} \bar{x}_{i j} \otimes w_{j}\right)^{\alpha} \\
=\left[\sum_{j=1}^{h}\left(s_{i j}-r_{i j}\right)\left(b_{j}-a_{j}\right) \alpha^{2}+\sum_{j=1}^{h}\left[r_{i j}\left(b_{j}-a_{j}\right)+a_{j}\left(s_{i j}-r_{i j}\right)\right] \alpha+\sum_{j=1}^{h} r_{i j} a_{j},\right. \\
\left.\sum_{j=1}^{h}\left(s_{i j}-t_{i j}\right)\left(b_{j}-c_{j}\right) \alpha^{2}+\sum_{j=1}^{h}\left[t_{i j}\left(b_{j}-c_{j}\right)+c_{j}\left(s_{i j}-t_{i j}\right)\right] \alpha+\sum_{j=1}^{h} t_{i j} c_{j}\right] \\
{\left[\sum_{j=h+1}^{n}\left(s_{i j}-r_{i j}\right)\left(b_{j}-a_{j}\right) \alpha^{2}+\sum_{j=h+1}^{n}\left[r_{i j}\left(b_{j}-a_{j}\right)+a_{j}\left(s_{i j}-r_{i j}\right)\right] \alpha+\sum_{j=h+1}^{n} r_{i j} a_{j},\right.} \\
\left.\sum_{j=h+1}^{n}\left(s_{i j}-t_{i j}\right)\left(b_{j}-c_{j}\right) \alpha^{2}+\sum_{j=h+1}^{n}\left[t_{i j}\left(b_{j}-c_{j}\right)+c_{j}\left(s_{i j}-t_{i j}\right)\right] \alpha+\sum_{j=h+1}^{n} t_{i j} c_{j}\right] \\
\quad E_{i 1}=\sum_{j=1}^{h}\left(s_{i j}-r_{i j}\right)\left(b_{j}-a_{j}\right), F_{i 1}=\sum_{j=1}^{h}\left[r_{i j}\left(b_{j}-a_{j}\right)+a_{j}\left(s_{i j}-r_{i j}\right)\right], \\
E_{i 2}=\sum_{j=1}^{h}\left(s_{i j}-t_{i j}\right)\left(b_{j}-c_{j}\right), F_{i 2}=\sum_{j=1}^{h}\left[t_{i j}\left(b_{j}-c_{j}\right)+c_{j}\left(s_{i j}-t_{i j}\right)\right], \\
I_{i 1}=\sum_{j=h+1}^{n}\left(s_{i j}-r_{i j}\right)\left(b_{j}-a_{j}\right), J_{i 1}=\sum_{j=h+1}^{n}\left[r_{i j}\left(b_{j}-a_{j}\right)+a_{j}\left(s_{i j}-r_{i j}\right)\right],
\end{gathered}
$$




$$
\begin{gathered}
I_{i 2}=\sum_{j=h+1}^{n}\left(s_{i j}-t_{i j}\right)\left(b_{j}-c_{j}\right), J_{i 2}=\sum_{j=h+1}^{n}\left[t_{i j}\left(b_{j}-c_{j}\right)+c_{j}\left(s_{i j}-t_{i j}\right)\right], \\
V_{i 1}=\sum_{j=1}^{h} r_{i j} a_{j}, Y_{i 1}=\sum_{j=1}^{h} s_{i j} b_{j}, Z_{i 1}=\sum_{j=1}^{h} t_{i j} c_{j}, V_{i 2}=\sum_{j=h+1}^{n} r_{i j} a_{j}, \\
Y_{i 2}=\sum_{j=h+1}^{n} s_{i j} b_{j}, Z_{i 2}=\sum_{j=h+1}^{n} t_{i j} c_{j} .
\end{gathered}
$$

We now have two simplified equations to solve, namely:

$$
\begin{aligned}
& \left(E_{i 1}-I_{i 2}\right) \alpha^{2}+\left(F_{i 1}-J_{i 2}\right) \alpha+\left(V_{i 1}-Z_{i 2}\right)-x=0 \\
& \left(E_{i 2}-I_{i 1}\right) \alpha^{2}+\left(F_{i 2}-J_{i 1}\right) \alpha+\left(Z_{i 1}-V_{i 2}\right)-x=0
\end{aligned}
$$

Only roots in $[0,1]$ will be retained in (14) and (15). The left membership function, i.e. $f_{G_{i}}^{\mathrm{L}}(x)$, and the right membership function, i.e. $f_{G_{i}}^{\mathrm{R}}(x)$, of $G_{i}$ can then be produced as:

$$
\begin{gathered}
f_{G_{i}}^{\mathrm{L}}(x)=\left\{\left(J_{i 2}-F_{i 1}\right)+\left[\left(F_{i 1}-J_{i 2}\right)^{2}+4\left(E_{i 1}-I_{i 2}\right)\left(x-V_{i 1}+Z_{i 2}\right)\right]^{1 / 2}\right\} / 2\left(E_{i 1}-I_{i 2}\right), \\
V_{i 1}-Z_{i 2} \leq x \leq Y_{i 1}-Y_{i 2}, \\
f_{G_{i}}^{\mathrm{R}}(x)=\left\{\left(J_{i 1}-F_{i 2}\right)-\left[\left(F_{i 2}-J_{i 1}\right)^{2}+4\left(E_{i 2}-I_{i 1}\right)\left(x-Z_{i 1}+V_{i 2}\right)\right]^{1 / 2}\right\} / 2\left(E_{i 2}-I_{i 1}\right), \\
Y_{i 1}-Y_{i 2} \leq x \leq Z_{i 1}-V_{i 2} .
\end{gathered}
$$

For convenience, $G_{i}, i=1,2, \ldots, m$, can be expressed as:

$$
G_{i}=\left(V_{i 1}-Z_{i 2}, Y_{i 1}-Y_{i 2}, Z_{i 1}-V_{i 2} ; J_{i 2}-F_{i 1}, E_{i 1}-I_{i 2} ; J_{i 1}-F_{i 2}, E_{i 2}-I_{i 1}\right)
$$

Proposition 1. For Eq. (16), $f_{G_{i}}^{\mathrm{L}}(x)=0$ if $x=V_{i 1}-Z_{i 2}$.

\section{Proof.}

$$
\begin{gathered}
f_{G_{i}}^{\mathrm{L}}(x) \\
=\left\{\left(J_{i 2}-F_{i 1}\right)+\left[\left(F_{i 1}-J_{i 2}\right)^{2}+4\left(E_{i 1}-I_{i 2}\right)\left(x-V_{i 1}+Z_{i 2}\right)\right]^{1 / 2}\right\} / 2\left(E_{i 1}-I_{i 2}\right) \\
=\left\{\left(J_{i 2}-F_{i 1}\right)+\left[\left(F_{i 1}-J_{i 2}\right)^{2}+4\left(E_{i 1}-I_{i 2}\right)\left(V_{i 1}-Z_{i 2}-V_{i 1}+Z_{i 2}\right)\right]^{1 / 2}\right\} / 2\left(E_{i 1}-I_{i 2}\right) \\
=\left\{\left(J_{i 2}-F_{i 1}\right)+\left[\left(F_{i 1}-J_{i 2}\right)^{2}\right]^{1 / 2}\right\} / 2\left(E_{i 1}-I_{i 2}\right)=0 .
\end{gathered}
$$

Proposition 2. In Eq. (16), $E_{i 1}-I_{i 2}-J_{i 2}+F_{i 1}=Y_{i 1}-Y_{i 2}-V_{i 1}+Z_{i 2}$.

\section{Proof.}

$$
E_{i 1}-I_{i 2}-J_{i 2}+F_{i 1}
$$




$$
\begin{gathered}
=\sum_{j=1}^{h}\left(s_{i j}-r_{i j}\right)\left(b_{j}-a_{j}\right)-\sum_{j=h+1}^{n}\left(s_{i j}-t_{i j}\right)\left(b_{j}-c_{j}\right) \\
-\sum_{j=h+1}^{n}\left[t_{i j}\left(b_{j}-c_{j}\right)+c_{j}\left(s_{i j}-t_{i j}\right)\right]+\sum_{j=1}^{h}\left[r_{i j}\left(b_{j}-a_{j}\right)+a_{j}\left(s_{i j}-r_{i j}\right)\right] \\
=\sum_{j=1}^{h} s_{i j} b_{j}-\sum_{j=1}^{h} r_{i j} b_{j}-\sum_{j=1}^{h} s_{i j} a_{j}+\sum_{j=1}^{h} r_{i j} a_{j}-\sum_{j=h+1}^{n} s_{i j} b_{j}+\sum_{j=h+1}^{n} t_{i j} b_{j}+\sum_{j=h+1}^{n} s_{i j} c_{j}-\sum_{j=h+1}^{n} t_{i j} c_{j} \\
-\sum_{j=h+1}^{n} t_{i j} b_{j}+\sum_{j=h+1}^{n} t_{i j} c_{j}-\sum_{j=h+1}^{n} s_{i j} c_{j}+\sum_{j=h+1}^{n} t_{i j} c_{j}+\sum_{j=1}^{h} r_{i j} b_{j}-\sum_{j=1}^{h} r_{i j} a_{j}+\sum_{j=1}^{h} s_{i j} a_{j}-\sum_{j=1}^{h} r_{i j} a_{j} \\
=\sum_{j=1}^{h} s_{i j} b_{j}-\sum_{j=h+1}^{n} s_{i j} b_{j}-\sum_{j=1}^{h} r_{i j} a_{j}+\sum_{j=h+1}^{n} t_{i j} c_{j}=Y_{i 1}-Y_{i 2}-V_{i 1}+Z_{i 2} . \square
\end{gathered}
$$

Corollary 2.1. For Eq. (16), $f_{G_{i}}^{\mathrm{L}}(x)=1$ if $x=Y_{i 1}-Y_{i 2}$.

Proof.

$$
\begin{gathered}
f_{G_{i}}^{\mathrm{L}}(x) \\
=\left\{\left(J_{i 2}-F_{i 1}\right)+\left[\left(F_{i 1}-J_{i 2}\right)^{2}+4\left(E_{i 1}-I_{i 2}\right)\left(x-V_{i 1}+Z_{i 2}\right)\right]^{1 / 2}\right\} / 2\left(E_{i 1}-I_{i 2}\right) \\
=\left\{\left(J_{i 2}-F_{i 1}\right)+\left[\left(F_{i 1}-J_{i 2}\right)^{2}+4\left(E_{i 1}-I_{i 2}\right)\left(Y_{i 1}-Y_{i 2}-V_{i 1}+Z_{i 2}\right)\right]^{1 / 2}\right\} / 2\left(E_{i 1}-I_{i 2}\right)
\end{gathered}
$$

Proposition 2 obtains:

$$
f_{G_{i}}^{\mathrm{L}}(x)
$$

$$
\begin{gathered}
=\left\{\left(J_{i 2}-F_{i 1}\right)+\left[\left(F_{i 1}-J_{i 2}\right)^{2}+4\left(E_{i 1}-I_{i 2}\right)\left(E_{i 1}-I_{i 2}-J_{i 2}+F_{i 1}\right)\right]^{1 / 2}\right\} / 2\left(E_{i 1}-I_{i 2}\right) \\
=\left\{\left(J_{i 2}-F_{i 1}\right)+\left[\left(F_{i 1}-J_{i 2}\right)^{2}+4\left(E_{i 1}-I_{i 2}\right)^{2}+4\left(E_{i 1}-I_{i 2}\right)\left(F_{i 1}-J_{i 2}\right)\right]^{1 / 2}\right\} / 2\left(E_{i 1}-I_{i 2}\right) \\
=\left\{\left(J_{i 2}-F_{i 1}\right)+\left[\left(2\left(E_{i 1}-I_{i 2}\right)+\left(F_{i 1}-J_{i 2}\right)\right)^{2}\right]^{1 / 2}\right\} / 2\left(E_{i 1}-I_{i 2}\right)=1 .
\end{gathered}
$$

Proposition 3. In Eq. (17), $F_{i 2}-J_{i 1}+E_{i 2}-I_{i 1}=Y_{i 1}-Y_{i 2}-Z_{i 1}+V_{i 2}$.

Proof.

$$
\begin{gathered}
F_{i 2}-J_{i 1}+E_{i 2}-I_{i 1} \\
=\sum_{j=1}^{h}\left[t_{i j}\left(b_{j}-c_{j}\right)+c_{j}\left(s_{i j}-t_{i j}\right)\right]-\sum_{j=h+1}^{n}\left[r_{i j}\left(b_{j}-a_{j}\right)+a_{j}\left(s_{i j}-r_{i j}\right)\right] \\
+\sum_{j=1}^{h}\left(s_{i j}-t_{i j}\right)\left(b_{j}-c_{j}\right)-\sum_{j=h+1}^{n}\left(s_{i j}-r_{i j}\right)\left(b_{j}-a_{j}\right)
\end{gathered}
$$




$$
\begin{gathered}
=\sum_{j=1}^{h} t_{i j} b_{j}-\sum_{j=1}^{h} t_{i j} c_{j}+\sum_{j=1}^{h} s_{i j} c_{j}-\sum_{j=1}^{h} t_{i j} c_{j}-\sum_{j=h+1}^{n} r_{i j} b_{j}+\sum_{j=h+1}^{n} r_{i j} a_{j}-\sum_{j=h+1}^{n} s_{i j} a_{j}+\sum_{j=h+1}^{n} r_{i j} a_{j} \\
+\sum_{j=1}^{h} s_{i j} b_{j}-\sum_{j=1}^{h} t_{i j} b_{j}-\sum_{j=1}^{h} s_{i j} c_{j}+\sum_{j=1}^{h} t_{i j} c_{j}-\sum_{j=h+1}^{n} s_{i j} b_{j}+\sum_{j=h+1}^{n} r_{i j} b_{j}+\sum_{j=h+1}^{n} s_{i j} a_{j}-\sum_{j=h+1}^{n} r_{i j} a_{j} \\
=\sum_{j=1}^{h} s_{i j} b_{j}-\sum_{j=h+1}^{n} s_{i j} b_{j}-\sum_{j=1}^{h} t_{i j} c_{j}+\sum_{j=h+1}^{n} r_{i j} a_{j}=Y_{i 1}-Y_{i 2}-Z_{i 1}+V_{i 2} .
\end{gathered}
$$

Corollary 3.1. For Eq. (17), $f_{G_{i}}^{\mathrm{R}}(x)=1$ if $x=Y_{i 1}-Y_{i 2}$.

Proof.

$$
\begin{gathered}
f_{G_{i}}^{\mathrm{R}}(x) \\
=\left\{\left(J_{i 1}-F_{i 2}\right)-\left[\left(F_{i 2}-J_{i 1}\right)^{2}+4\left(E_{i 2}-I_{i 1}\right)\left(x-Z_{i 1}+V_{i 2}\right)\right]^{1 / 2}\right\} / 2\left(E_{i 2}-I_{i 1}\right) \\
=\left\{\left(J_{i 1}-F_{i 2}\right)-\left[\left(F_{i 2}-J_{i 1}\right)^{2}+4\left(E_{i 2}-I_{i 1}\right)\left(Y_{i 1}-Y_{i 2}-Z_{i 1}+V_{i 2}\right)\right]^{1 / 2}\right\} / 2\left(E_{i 2}-I_{i 1}\right)
\end{gathered}
$$

Proposition 3 produces:

$$
\begin{gathered}
f_{G_{i}}^{\mathrm{R}}(x) \\
=\left\{\left(J_{i 1}-F_{i 2}\right)-\left[\left(F_{i 2}-J_{i 1}\right)^{2}+4\left(E_{i 2}-I_{i 1}\right)\left(F_{i 2}-J_{i 1}+E_{i 2}-I_{i 1}\right)\right]^{1 / 2}\right\} / 2\left(E_{i 2}-I_{i 1}\right) \\
=\left\{\left(J_{i 1}-F_{i 2}\right)-\left[\left(F_{i 2}-J_{i 1}\right)^{2}+4\left(E_{i 2}-I_{i 1}\right)\left(F_{i 2}-J_{i 1}\right)+4\left(E_{i 2}-I_{i 1}\right)^{2}\right]^{1 / 2}\right\} / 2\left(E_{i 2}-I_{i 1}\right) \\
=\left\{\left(J_{i 1}-F_{i 2}\right)-\left[\left(2\left(E_{i 2}-I_{i 1}\right)+\left(F_{i 2}-J_{i 1}\right)\right)^{2}\right]^{1 / 2}\right\} / 2\left(E_{i 2}-I_{i 1}\right) \\
=\left\{\left(J_{i 1}-F_{i 2}\right)-\left[\left(2\left(I_{i 1}-E_{i 2}\right)+\left(J_{i 1}-F_{i 2}\right)\right)^{2}\right]^{1 / 2}\right\} / 2\left(E_{i 2}-I_{i 1}\right)=1 .
\end{gathered}
$$

Proposition 4. For Eq. (17), $f_{G_{i}}^{\mathrm{R}}(x)=0$ if $x=Z_{i 1}-V_{i 2}$.

Proof.

$$
\begin{gathered}
f_{G_{i}}^{\mathrm{R}}(x) \\
=\left\{\left(J_{i 1}-F_{i 2}\right)-\left[\left(F_{i 2}-J_{i 1}\right)^{2}+4\left(E_{i 2}-I_{i 1}\right)\left(x-Z_{i 1}+V_{i 2}\right)\right]^{1 / 2}\right\} / 2\left(E_{i 2}-I_{i 1}\right) \\
=\left\{\left(J_{i 1}-F_{i 2}\right)-\left[\left(F_{i 2}-J_{i 1}\right)^{2}+4\left(E_{i 2}-I_{i 1}\right)\left(Z_{i 1}-V_{i 2}-Z_{i 1}+V_{i 2}\right)\right]^{1 / 2}\right\} / 2\left(E_{i 2}-I_{i 1}\right) \\
=\left\{\left(J_{i 1}-F_{i 2}\right)-\left[\left(-F_{i 2}+J_{i 1}\right)^{2}\right]^{1 / 2}\right\} / 2\left(E_{i 2}-I_{i 1}\right)=0 .
\end{gathered}
$$




\subsection{Determine the Ordering of Alternatives}

Herein, an intuitive ranking method, the centroid index (Yager, 1981) is applied to help rank all the final fuzzy numbers.

Suppose fuzzy numbers $A_{i}, A_{i}=\left[a_{i}, b_{i}, c_{i}, d_{i}\right]$, as in Definition 1, with membership function $f_{A_{i}}(x)$. The centroid of $A, C\left(A_{i}\right)$, on the horizontal axis can be defined as:

$$
C\left(A_{i}\right)=\frac{\int_{a_{i}}^{b_{i}}\left(x f_{A_{i}}^{\mathrm{L}}\right) \mathrm{d} x+\int_{b_{i}}^{c_{i}} x \mathrm{dx}+\int_{c_{i}}^{d_{i}}\left(x f_{A_{i}}^{\mathrm{R}}\right) \mathrm{d} x}{\int_{a_{i}}^{b_{i}}\left(f_{A_{i}}^{\mathrm{L}}\right) \mathrm{d} x+\int_{b_{i}}^{c_{i}} \mathrm{~d} x+\int_{c_{i}}^{d_{i}}\left(f_{A_{i}}^{\mathrm{R}}\right) \mathrm{d} x}
$$

According to the proposed concept, the smaller the $C\left(A_{i}\right)$, the higher priority the alternative $A_{i}$ will have. Thus, for any two final fuzzy numbers $G_{i}$ and $G_{j}$, if $C\left(G_{i}\right)<C\left(G_{j}\right)$, then $G_{i}>G_{j}$. If $C\left(G_{i}\right)=C\left(G_{j}\right)$, then $G_{i}=G_{j}$. Finally, if $C\left(G_{i}\right)>C\left(G_{j}\right)$, then $G_{i}<G_{j}$.

\section{Numerical Example}

Assume that a manufacturing company needs a robot to perform a material-handling task (Liang and Wang, 1993). After preliminary screening, three robots $R_{1}, R_{2}$ and $R_{3}$ are chosen for further evaluation. A committee of three decision-makers $D_{1}, D_{2}$ and $D_{3}$ is formed to conduct the evaluation and selection of the three robots. Two cost criteria, purchase cost $\left(C_{1}\right)$ and positioning accuracy $\left(C_{2}\right)$, and two benefit criteria, programming flexibility $\left(C_{3}\right)$ and man-machine interface $\left(C_{4}\right)$, are considered.

Further assume that the decision-makers use the linguistic rating set $S=\{\mathrm{VP}, \mathrm{P}, \mathrm{F}, \mathrm{G}, \mathrm{VG}\}$, where $\mathrm{VP}=$ Very Poor $=(0,0,3), \quad \mathrm{P}=$ Poor $=(0,3,5), \quad \mathrm{F}=\mathrm{Fair}=(2,5,8)$, $\mathrm{G}=\mathrm{Good}=(5,7,10)$, and $\mathrm{VG}=$ Very $\operatorname{Good}=(7,10,10)$ to evaluate the suitability of each robot under each of benefit criteria. And the decision-makers employ a linguistic weighting set $W=\{\mathrm{VL}, \mathrm{L}, \mathrm{M}, \mathrm{H}, \mathrm{VH}\}$, where $\mathrm{VL}=$ Very Low $=(0,0.1,0.3), \mathrm{L}=\mathrm{Low}=(0.1,0.3,0.5), \mathrm{M}=$ Medium $=(0.3,0.5$ ,0.7) $\mathrm{H}=\mathrm{High}=(0.5,0.7,0.9)$, and $\mathrm{VH}=\operatorname{Very} \operatorname{High}=(0.7,0.9,1)$, to assess the importance of all the criteria. Moreover, assume that the cost (million NT\$) and positioning accuracy ( \pm in.) assessed by decision-makers for the three robots are presented in Table 1 and the suitability ratings of three robots versus benefit criteria are also shown in Table 1 . The importance weights of the criteria are displayed in Table 2.

Table 1. Ratings of robots, averaged ratings and normalized ratings

\begin{tabular}{|c|c|c|c|c|c|c|}
\hline \multirow{2}{*}{ C. } & \multirow{2}{*}{ R. } & \multicolumn{3}{|c|}{ Decision-makers } & \multirow{2}{*}{ Averaged ratings $x_{i j}$} & \multirow{2}{*}{ Normalized ratings $\bar{x}_{i j}$} \\
\hline & & $\mathrm{D}_{1}$ & $\mathrm{D}_{2}$ & $\mathrm{D}_{3}$ & & \\
\hline \multirow{3}{*}{$C_{1}$} & $\mathrm{R}_{1}$ & \multicolumn{3}{|c|}{$(3.1,3.5,3.7)$} & $(3.1,3.5,3.7)$ & $(0.7561,0.8537,0.9024)$ \\
\hline & $R_{2}$ & \multicolumn{3}{|c|}{$(3.3,3.5,3.8)$} & $(3.3,3.5,3.8)$ & $(0.8049,0.8537,0.9268)$ \\
\hline & $R_{3}$ & \multicolumn{3}{|c|}{$(3.4,3.7,4.1)$} & $(3.4,3.7,4.1)$ & $(0.8293,0.9024,1.0000)$ \\
\hline \multirow{3}{*}{$C_{2}$} & $\mathrm{R}_{1}$ & \multicolumn{3}{|c|}{$(0.10,0.13,0.14)$} & $(0.10,0.13,0.14)$ & $(0.5882,0.7647,0.8235)$ \\
\hline & $R_{2}$ & \multicolumn{3}{|c|}{$(0.09,0.13,0.17)$} & $(0.09,0.13,0.17)$ & $(0.5294,0.7647,1.0000)$ \\
\hline & $R_{3}$ & \multicolumn{3}{|c|}{$(0.09,0.12,0.14)$} & $(0.09,0.12,0.14)$ & $(0.5294,0.7059,0.8235)$ \\
\hline \multirow{3}{*}{$C_{3}$} & $\mathrm{R}_{1}$ & VG & VG & $\mathrm{G}$ & $(6.3333,9.0000,10.0000)$ & $(0.6333,0.9000,1.0000)$ \\
\hline & $R_{2}$ & $\mathrm{G}$ & G & $\mathrm{G}$ & $(5.0000,7.0000,10.0000)$ & $(0.5000,0.7000,1.0000)$ \\
\hline & $R_{3}$ & $\mathrm{G}$ & VG & VG & $(6.3333,9.0000,10.0000)$ & $(0.6333,0.9000,1.0000)$ \\
\hline \multirow{3}{*}{$C_{4}$} & $\mathrm{R}_{1}$ & $\mathrm{~F}$ & G & $\mathrm{G}$ & $(4.0000,6.3333,9.3333)$ & $(0.4286,0.6786,1.0000)$ \\
\hline & $R_{2}$ & G & $\mathrm{F}$ & G & $(4.0000,6.3333,9.3333)$ & $(0.4286,0.6786,1.0000)$ \\
\hline & $R_{3}$ & $\mathrm{~F}$ & $\mathrm{G}$ & $\mathrm{F}$ & $(3.0000,5.6667,8.6667)$ & $(0.3214,0.6071,0.9286)$ \\
\hline
\end{tabular}

C.: Criteria, R.: Robots.

By Eqs. (9) and (10), the averaged rating and normalized averaged rating of each robot $R_{i}$ under each criterion from the decision-making committee can be obtained as also shown in Table 1. By Eq. (11), the averaged weights of the criteria from the decision-making committee can be obtained as also shown in Table 2.

Table 2. The weights of criteria and averaged weights

\begin{tabular}{lcccc}
\hline & \multicolumn{3}{c}{ Decision-makers } & \\
\cline { 2 - 4 } $\mathrm{C}$. & $D_{1}$ & $D_{2}$ & $D_{3}$ & Averaged weights $w_{j}$ \\
\hline$C_{1}$ & $\mathrm{H}$ & $\mathrm{H}$ & $\mathrm{VH}$ & $(0.5667,0.7667,0.9333)$ \\
$C_{2}$ & $\mathrm{VH}$ & $\mathrm{VH}$ & $\mathrm{H}$ & $(0.6333,0.8333,0.9667)$ \\
$C_{3}$ & $\mathrm{H}$ & $\mathrm{M}$ & $\mathrm{H}$ & $(0.4333,0.6333,0.8333)$ \\
$C_{4}$ & $\mathrm{M}$ & $\mathrm{L}$ & $\mathrm{L}$ & $(0.1667,0.3667,0.5667)$ \\
\hline
\end{tabular}

C.: Criteria. 
By Eqs. (12)-(18), the membership function for $G_{i}$, $i=1 \sim 3$, can be obtained as follows:

$G_{1}=(-0.5900,0.4729,1.2925 ;-1.1014,-0.0295$;

$0.7322,-0.0874)$,

$G_{2}=(-0.6086,0.5996,1.5436 ;-1.2757,-0.0675$;

$0.8976,-0.0464)$,

$G_{3}=(-0.5543,0.4875,1.4014 ;-1.0762,-0.0344$;

$0.8354,-0.0785)$.

Finally, Eq. (19) produces $C\left(G_{1}\right)=0.3988, C\left(G_{2}\right)$ $=0.5210$ and $C\left(G_{3}\right)=0.4543$. Obviously, the ranking order of the three robots is $R_{1}>R_{3}>R_{2}$. Thus, the best selection is robot $R_{1}$.

\section{Conclusions}

This work suggests a new fuzzy MCDM algorithm, which is established based on subtracting the summation of weighted normalized benefit ratings from that of weighted normalized cost ratings for each alternative. Using interval arithmetic of fuzzy numbers can then clearly develop the membership function of the final aggregation of fuzzy numbers. The centroid method of fuzzy number ranking has been applied to help determine ordering of all alternatives.

The suggested method provides an extension to the fuzzy MCDM techniques available. A numerical example has demonstrated feasibility and computational process of the proposed method.

\section{Acknowledgements}

This work was supported in part by Ministry of Science and Technology, Taiwan, ROC, under Grant MOST 103-2410-H-218-008-MY2.

\section{REFERENCES}

[1] Afkham, L., Abdi, F. and Rashidi, A. (2012), Evaluation of Service Quality by Using Fuzzy MCDM: A Case Study in Iranian Health-Care Centers, Management Science Letters, 2(1), 291-300.

[2] Akdag, H., Kalayc, T., Karagöz, S., Zülfikar, H. \& Giz, D. (2014), The Evaluation of Hospital Service Quality by Fuzzy MCDM, Applied Soft Computing, 23, 239-248.

[3] Asady, B. (2010), The Revised Method of Ranking LR Fuzzy Number Based on Deviation Degree, Expert Systems with Applications, 37(7), 5056-5060.

[4] Büyüközkan, G., Feyzioglu, O. and Cifçi, G. (2011), Fuzzy Multi-Criteria Evaluation of Knowledge Management Tools, International Journal of Computational Intelligence Systems, 4(2), 184-195.

[5] Carlsson, C. and Fuller, R. (1996), Fuzzy Multiple Criteria
Decision Making: Recent Developments, Fuzzy Sets and Systems 78 (2), 139-153.

[6] Chen, C.T. (2001), A Fuzzy Approach to Select the Location of the Distribution Center, Fuzzy Sets and Systems 118 (1), 65-73.

[7] Chen, S.J. and Hwang, C.L. (1992), Fuzzy Multiple Attribute Decision Making, Berlin: Springer.

[8] Chu, T.C. and Varma, R. (2012), Evaluating Suppliers via a Multiple Levels Multiple Criteria Decision Making Method under Fuzzy Environment, Computer and Industrial Engineering 62 (2), 653-660.

[9] Chung, Y.F., Liu, S.H., Wang, C.H. and Pang, C.T. (2015), Applying Fuzzy MCDM Methods to the Evaluation on Portal Website Service Quality, The SIJ Transactions on Computer Science Engineering \& its Applications (CSEA), 3(1), 8-15.

[10] Dubois, D. and Prade, H. (1978), Operations on Fuzzy Numbers, International Journal of Systems Science, 9(6), 613-626.

[11] Ezzati, R. Allahviranloo, T., Khezerloo, S. and Khezerloo, M. (2012), An Approach for Ranking of Fuzzy Number, Expert Systems with Applications, 39(1), 690-695.

[12] Ghorbani, M., Arabzad, S.M. and Shahin, A. (2013), A Novel Approach for Supplier Selection based on the Kano Model and Fuzzy MCDM, International Journal of Production Research, 51(18), 5469-5484.

[13] Govindan, K., Khodaverdi, R. and Jafarian, A. (2013), A Fuzzy Multi Criteria Approach for Measuring Sustainability Performance of a Supplier Based on Triple Bottom Line Approach, Journal of Cleaner Production, 47, 345-354.

[14] Hari Ganesh, A. \& Jayakumar, S. (2014). Ranking of Fuzzy Numbers Using Radius of Gyration of Centroids. International Journal of Basic and Applied Sciences, 3(1), $17-22$.

[15] Jafarian, E. and Rezvani, M. A. (2013), A Valuation-Based Method for Ranking the Intuitionistic Fuzzy Numbers, Journal of Intelligent \& Fuzzy Systems, 24(1), 133-144.

[16] Kaufmann, A. and Gupta, M. M. (1991), Introduction to Fuzzy Arithmetic: Theory and Application, New York: Van Nostrand Reinhold.

[17] Laarhoven, P.J.M. van and Pedrycz, W. (1983), A Fuzzy Extension of Saaty's Priority Theory, Fuzzy Sets and Systems, $11(1-3), 199-227$.

[18] Liang, G.S. and Wang, M. -J.J. (1993), A Fuzzy Multi-Criteria Decision-Making Approach for Robot Selection, Robotics \& Computer-Integrated Manufacturing, 10 (4), 267-274.

[19] Moghimi, R. and Anvari, A. (2014), An Integrated Fuzzy MCDM Approach and Analysis to Evaluate the Financial Performance of Iranian Cement Companies, The International Journal of Advanced Manufacturing Technology, 71 (1-4), 685-698.

[20] Rao, P.P.B. and Shankar, N.R. (2013), Ranking Fuzzy Numbers with an Area Method Using Circumcenter of Centroids, Fuzzy Information and Engineering, 5(1), 3-18

[21] Ribeiro, R.A. (1996), Fuzzy Mulitple Attribute Decision Making: A Review and New Preference Elicitation 
Techniques, Fuzzy Sets and Systems, 78 (2), 155-181.

[22] Sharma U. (2015), A New Lexicographical Approach for Ranking Fuzzy Numbers, Mathematical Theory and Modeling, 5(2), 143-152.

[23] Thorani, Y. L. P., Phani Bushan Rao, P. and Ravi Shankar, N. (2012), Ordering Generalized Trapezoidal Fuzzy Numbers, International Journal of Contemporary Mathematical Science, $7(12), 555-573$.

[24] Wang X. and Kerre, E. E. (2001), Reasonable Properties for the Ordering of Fuzzy Quantities (I) \& (II), Fuzzy Sets and Systems, 118(3), 375-385 and 387-405.
[25] Wang, Y.J. and Lee, H.S. (2008), The Revised Method of Ranking Fuzzy Numbers with an Area between the Centroid and Original Points, Computers \& Mathematics with Applications, 55(9), 2033-2042.

[26] Yager, R.R. (1981), A Procedure for Ordering Fuzzy Subsets of the Unit Interval, Information. Sciences, 24(2), 143-161.

[27] Zadeh, L. A. (1975), The Concept of a Linguistic Variable and Its Application to Approximate Reasoning, part 1, 2 and 3, Information Science, 8(3), 199-249, 8(4) 301-357, 9(1), 43-80.

[28] Zadeh, L. A. (1965), Fuzzy Sets, Information and Control, $8(3), 338-35$. 